\title{
Betacellulin inhibits amylase and glucagon production and promotes beta cell differentiation in mouse embryonic pancreas
}

\author{
S. Thowfeequ $\cdot$ K. L. Ralphs $\cdot$ W.-Y. Yu \\ J. M. W. Slack • D. Tosh
}

Received: 7 January 2007 / Accepted: 25 April 2007 / Published online: 12 June 2007

(C) Springer-Verlag 2007

\begin{abstract}
Aims/hypothesis Betacellulin, a member of the epidermal growth factor family, is expressed in the pancreas and is thought to regulate differentiation of beta cells during development. The aim of the present study was to investigate the effects of exogenous betacellulin on the development of the mouse embryonic pancreas.

Materials and methods We used an in vitro culture model system based on the isolation and culture of the dorsal embryonic pancreas from day 11.5 embryos. Cultures were treated for up to 10 days with $10 \mathrm{ng} / \mathrm{ml}$ betacellulin and then analysed for changes in the expression of pancreatic exocrine, endocrine and ductal markers.

Results Pancreases developed in culture and expressed the full complement of exocrine (both acinar and ductal) and endocrine cell types. Betacellulin enhanced branching morphogenesis and the proliferation of mesenchyme, increased $\mathrm{Pdx} 1$ and insulin production and inhibited the production of the exocrine cell marker amylase and the endocrine hormone glucagon.

Conclusions/interpretation These results suggest betacellulin has distinct and separate effects on exocrine, endocrine and ductal differentiation. In the future, betacellulin could perhaps be utilised to increase the production of beta cells from embryonic pancreatic tissue for therapeutic transplantation.
\end{abstract}

Electronic supplementary material The online version of this article (doi:10.1007/s00125-007-0724-y) contains supplementary material, which is available to authorised users.

S. Thowfeequ $\cdot$ K. L. Ralphs $\cdot$ W.-Y. Yu · J. M. W. Slack

D. Tosh $(\triangle)$

Centre for Regenerative Medicine, Department of Biology and Biochemistry, University of Bath,

Bath BA2 7AY, UK

e-mail: D.Tosh@bath.ac.uk
Keywords Amylase - Beta cell · Betacellulin · Cytokeratin . Ductal morphogenesis · Glucagon · Insulin · Islets · Pancreas
Abbreviations
CK19 cytokeratin 19
EGF epidermal growth factor
EGFR epidermal growth factor receptor
HNF hepatocyte nuclear factor
PH3 phosphohistone-3
PP pancreatic polypeptide
SS somatostatin
Stat3 signal transducer and activator of transcription 3

\section{Introduction}

Cell therapy for type 1 diabetes involves replacing the functioning beta cell complement of the body. Islet transplantation procedures have recently improved considerably but they still involve problems of immune rejection and donor supply [1,2]. One way forward is to augment the supply of pancreatic tissue for transplantation by producing beta cells in vitro. Recent progress in stem cell biology has demonstrated the generation of insulin-expressing cells from different sources including embryonic stem cells [3] and fetal hepatocytes [4].

Advances in our understanding of the developmental biology of the pancreas will be useful in producing an alternative supply of beta cells. For example, identification of growth factors that suppress exocrine development while enhancing the endocrine beta cell compartment might be useful in producing an alternative supply of tissue for transplantation.

Signalling through the ErbB receptors regulates lineage determination during pancreatic development [5]. The 
ErbB family of transmembrane receptor tyrosine kinases encompasses four members (ErbB1 or epidermal growth factor [EGF] receptor [EGFR], ErbB2 or Neu, ErbB3 and ErbB4) that bind to similar ligands and maintain interreceptor interactions through ligand-induced homodimerisation and heterodimerisation [6]. An increasing number of growth factors are being discovered that serve as ligands for the ErbB receptor family. In mammals they include EGF, HB-EGF, epiregulin, amphiregulin, the neuregulin subfamily and betacellulin, all sharing high sequence similarity with a six-cysteine 36-40 amino acid residue EGF motif [6].

Betacellulin was isolated as a $32 \mathrm{kDa}$ glycoprotein from the conditioned medium of a mouse pancreatic insulinoma cell line and signals through both ErbB1 and ErbB4 receptors $[7,8]$. The expression pattern of betacellulin is widespread, with particularly high expression in the pancreas, liver and kidneys. In the fetal pancreas, betacellulin has been immunohistochemically localised to the primitive duct cells and alpha cells and probably beta cells [8]. Betacellulin knockout mice show no obvious pancreatic phenotype [9].

The biological function of betacellulin has mostly been defined by its effects on cultured cell lines in vitro. Early studies using the AR42J-B13 cell line showed that betacellulin and activin A can convert these amylase-secreting cells to insulin-expressing cells [10]. Later Demeterco et al. [11] demonstrated that betacellulin has a mitogenic effect on primary cultures of undifferentiated human fetal pancreatic epithelial cells but it does not induce these cell types to differentiate along the endocrine pathway. The combination of betacellulin and activin $\mathrm{A}$ induced regeneration of beta cells in neonatal streptozotocin (STZ)-treated rats and consequently improved glucose metabolism [12]. Betacellulin has been used in combination with NeuroD to trigger formation of insulin-secreting cells in the liver and completely reverse diabetes in STZ-treated mice [13]. Intraperitoneal injection of betacellulin boosts beta cell regeneration in 90\%-pancreatectomised Wistar rats [14]. Betacellulin also facilitates cell proliferation and tubulogenesis during kidney and mammary gland development and promotes angiogenesis $[15,16]$. All this evidence suggests that betacellulin can have important effects on the development of the pancreas itself. Although betacellulin is not essential for pancreatic development, it may still exert some effects on application. To date the effects of exogenous betacellulin on embryonic pancreatic development have not been studied in detail. In the present paper we examined the effects of betacellulin on the morphogenesis of the dorsal pancreas and the formation of endocrine, exocrine and ductal cell types. We show that betacellulin enhances beta cell formation and ductal morphogenesis but inhibits formation of alpha cells and of exocrine cells.

\section{Materials and methods}

Dissection and culture of the embryonic pancreas All animal experiments were performed under UK Home Office regulations and with the approval of the University of Bath Ethics Committee. The pancreases were isolated and cultured as described previously [17, 18]. Further details on the isolation and culture are provided in the accompanying Electronic Supplementary Material (ESM). For treatments, $10 \mathrm{ng} / \mathrm{ml}$ of human recombinant betacellulin (Sigma, Poole, UK), EGF (Sigma) or TGF $\alpha$ (R\&D Systems, Abingdon, UK) was added to the culture medium.

RNA extraction and RT-PCR Freshly isolated pancreatic buds from 11.5-day (E11.5d) embryos were treated with Eagle's Minimal Essential Medium with Hanks' salts containing $10 \%$ (vol./vol.) fetal bovine serum and $1 \mathrm{mg} / \mathrm{ml}$ type 1A collagenase (all Sigma) for $8 \mathrm{~min}$ at room temperature. The buds were washed in PBS and then the mesenchymal tissue was separated from the more opaque epithelial tissue using tungsten needles. Total RNA was then extracted from the isolated mesenchyme or epithelium from 30 pancreatic buds using TRI reagent (Sigma) according to the manufacturer's instructions. Aliquots $(3 \mu \mathrm{g})$ of RNA were treated with DNase I and reversed transcription was carried out as previously described [19]. Of the cDNA, $75 \mathrm{ng}$ were amplified by PCR in $1.1 \times$ ReddyMix PCR Master Mix (ABgene, Epsom, UK) containing $50 \mathrm{ng}$ of each primer pair. Details of the mouse primers [20] are found in the accompanying ESM.

Immunohistochemistry Pancreases were fixed in either MEMFA (3.8\% [vol./vol.] formaldehyde, $0.15 \mathrm{~mol} / 1 \mathrm{MOPS}$, $2 \mathrm{mmol} / \mathrm{l}$ EGTA, $1 \mathrm{mmol} / \mathrm{l} \mathrm{MgSO}_{4}, \mathrm{pH} 7.4$ ) at room temperature for $20 \mathrm{~min}$ or acetone:methanol (1:1) for $5 \mathrm{~min}$ at $-20^{\circ} \mathrm{C}$. Antigen retrieval was performed on samples for staining of cytokeratin-19 (CK19) and E-cadherin by treating the acetone:methanol-fixed tissue with citrate buffer (Lab Vision, Newmarket, UK) for $1 \mathrm{~h}$ at $37^{\circ} \mathrm{C}$. For a more detailed description of the immunostaining see the ESM.

Protein extraction, quantification and western blotting analysis Western blotting was performed by conventional techniques as described in the ESM.

Microscopy and photography During the culture period, the live samples were observed using a Leica DMIRB inverted microscope (Leica, Milton Keynes, UK) and the immunostained samples were visualised under a Leica DMRB compound microscope. The images were taken using a colour SPOT RT camera (Diagnostic Instruments, Sterling Heights, MI, USA) operated with Advanced Spot 
RT 3.0 software. A Zeiss LSM510 confocal microscope (Carl Zeiss, Welwyn Garden City, UK) was used to take high-resolution images. The images were cropped and arranged using Adobe Photoshop 7.0. Typical results are shown for each treatment.

Measurements and statistics All experiments were repeated at least three times. The length of the ductal network of each pancreatic bud was measured as the sum of the lengths of individual branches from the base to the tip of the duct. The mean maximum diameter of beta cells was also calculated. Measurements of the length of branches and maximum diameter of cells and area measurements were performed using the overlay and measurement tools on the LSM510 Image Browser program. All the numerical results are presented as means \pm SEM. Significance of observed differences between different sample groups were tested by

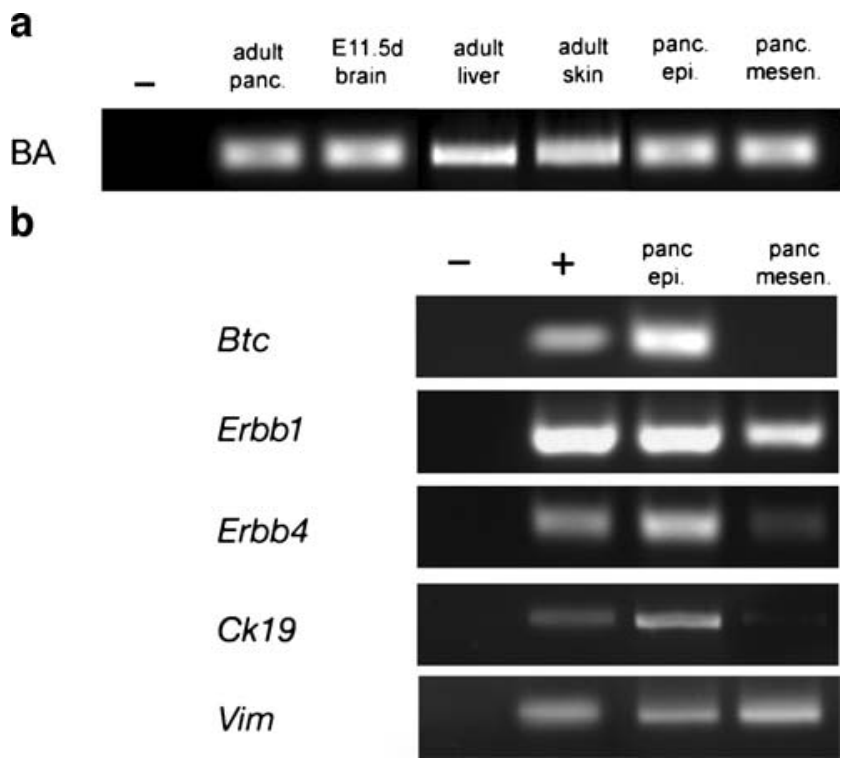

Fig. 1 a, b The expression of the genes for betacellulin (Btc) and the receptors ErbB1 (Erbb1, also known as Egfr) and ErbB4 (Erbb4) in the epithelium and mesenchyme of E11.5d mouse dorsal pancreas. a Shows the $\beta$-actin equal loading control for all the samples and the positive controls used. The - lane represents the water control (no cDNA). b Epithelium (panc epi.) and mesenchyme (panc mesen.) were separated as described in the "Materials and methods" section. Using PCR, we determined the expression of Btc, Erbbl and Erbb4 and the genes encoding CK19 (Ck19, also known as Krt19) and vimentin (Vim), markers of epithelium and mesenchyme, respectively, to demonstrate efficient separation of the two tissues. The results of the determination of $\mathrm{Ck} 19$ and Vim expression show that there is some contamination of the epithelium with mesenchyme but there is little contamination of the mesenchyme with epithelium. Btc expression is seen preferentially in the pancreatic epithelium. Expression of Erbb1 and Erbb4 is seen in both the pancreatic epithelium and mesenchyme, but at much higher levels in the epithelium. cDNA from adult pancreas and E11.5d embryonic brain were used as positive controls $(+)$ for $B t c$ and the $E r b B$ genes, respectively, and cDNA from liver and skin were positive controls for the epithelium $(\mathrm{Ck} 19)$ and mesenchyme (Vim), respectively a Mann-Whitney $U$ test performed using GraphPad Prism 3.0 software (GraphPad Software, San Diego, CA, USA).

\section{Results}

Differentiation of E11.5d embryonic pancreatic buds in vitro We initially investigated the expression of endogenous betacellulin in E11.5d developing pancreas. The gene encoding betacellulin was expressed in the epithelium but not the mesenchyme (Fig. 1). The genes encoding ErbB1 and ErbB4, the receptors for betacellulin, were expressed in both the mesenchyme and the epithelium, suggesting that betacellulin could differentially affect both tissue types (Fig. 1). Pancreatic buds adhere to the fibronectin substrate within a few hours of isolation and gradually flatten out over the culture period. Mesenchymal cells spread rapidly out of the explants to form a monolayer of cells surrounding the epithelial clump in the centre. After 10 days of culture with betacellulin, pancreatic buds had a thicker and a wider area of mesenchyme in comparison with untreated buds. We measured the total area occupied by the epithelium and the mesenchyme using E-cadherin and smooth muscle actin as their respective markers. The mesenchymal:epithelial ratio for control and betacellulin-treated buds is shown in Table 1 . The results indicate that there was more than a 1.5 -fold increase in the mesenchymal:epithelial ratio after betacellulin treatment.

Table 1 Betacellulin regulates embryonic pancreatic development

\begin{tabular}{|c|c|c|c|}
\hline & Control & Betacellulin & $n$ \\
\hline Mesenchymal:epithelial ratio & $13.20 \pm 1.01$ & $21.73 \pm 1.12^{\mathrm{a}}$ & 3 \\
\hline $\begin{array}{l}\text { Length of the entire ductal } \\
\text { network }\left(\times 10^{3} \mu \mathrm{m}\right)\end{array}$ & $2.40 \pm 0.25$ & $8.43 \pm 0.53^{\mathrm{b}}$ & 4 \\
\hline $\begin{array}{l}\text { Total area of amylase-positive } \\
\text { cells }\left(\times 10^{3} \mu \mathrm{m}^{2}\right)\end{array}$ & $32.11 \pm 3.41$ & $1.73 \pm 0.45^{\mathrm{b}}$ & 4 \\
\hline $\begin{array}{l}\text { Total area of insulin-positive } \\
\text { cells }\left(\times 10^{3} \mu \mathrm{m}^{2}\right)\end{array}$ & $72.75 \pm 8.26$ & $235.60 \pm 29.34^{\mathrm{a}}$ & 6 \\
\hline $\begin{array}{l}\text { Area of insulin-positive cells/ } \\
\text { no. of SS cells }\left(\mu \mathrm{m}^{2}\right)\end{array}$ & $439.7 \pm 43.24$ & $398.0 \pm 33.59^{\mathrm{c}}$ & 4 \\
\hline $\begin{array}{l}\text { Area of insulin-positive cells/ } \\
\text { no. of PP cells }\left(\mu^{2}\right)\end{array}$ & $578.8 \pm 50.47$ & $1,026.0 \pm 132.00^{\mathrm{a}}$ & 4 \\
\hline $\begin{array}{l}\text { Area of insulin-positive cells/ } \\
\text { no. of ghrelin cells }\left(\mu \mathrm{m}^{2}\right)\end{array}$ & $3,490 \pm 215.80$ & $1,937 \pm 224.50^{\mathrm{a}}$ & 4 \\
\hline Total PH3-positive cell count & $1,377 \pm 72.26$ & $2,165 \pm 115.10^{\mathrm{a}}$ & 4 \\
\hline $\begin{array}{l}\text { Maximum diameter of beta } \\
\text { cells }(\mu \mathrm{m})\end{array}$ & $13.91 \pm 0.24$ & $13.61 \pm 0.17^{\mathrm{c}}$ & 50 \\
\hline
\end{tabular}

Data are presented as the means \pm SEM.

${ }^{\mathrm{a}} p=0.0286$ vs control

${ }^{\mathrm{b}} p=0.0079$ vs control

${ }^{\mathrm{c}}$ Non-significant vs control 
Fig. 2 The effect of betacellulin on branching morphogenesis and amylase expression in the embryonic pancreas. Pancreatic buds were isolated from E11.5d embryos and cultured for $24 \mathrm{~h}$ to allow attachment. The medium was then replaced with fresh medium with and without betacellulin (10 ng/ml). Control (a, c, $\mathbf{e}, \mathbf{h}, \mathbf{j})$ and betacellulin-treated $(\mathbf{b}, \mathbf{d}, \mathbf{f}, \mathbf{g}, \mathbf{i}, \mathbf{k})$ samples are shown. After 9 days of treatment, the buds were fixed and immunostained. a, c Transmitted light images of control cultures. $\mathbf{b}, \mathbf{d}$ Transmitted lights images for betacellulin-treated cultures. At high magnification, the dense zymogen granules can be seen in the control cultures (c) but not in the betacellulin-treated cultures. e Immunostaining for CK19 in control cultures. f, g CK19 staining in betacellulin-treated cultures showing the elaborate branching network. h, i Immunostaining for the exocrine marker amylase in control (h) and betacellulin-treated (i) pancreatic buds. j, k Dual immunostaining for amylase (green) and insulin (red) in control (j) and betacellulin-treated $(\mathbf{k})$ cultures. $\mathbf{l}, \mathbf{m}$ The expression of activated caspase-3 in control (I) and betacellulin-treated samples (m). Scale bars: a, b, e, f, h, i $100 \mu \mathrm{m} ; \mathbf{c}, \mathbf{d}, \mathbf{g}, \mathbf{j}, \mathbf{k} 20 \mu \mathrm{m} ; \mathbf{l}, \mathbf{m}$ $10 \mu \mathrm{m}$
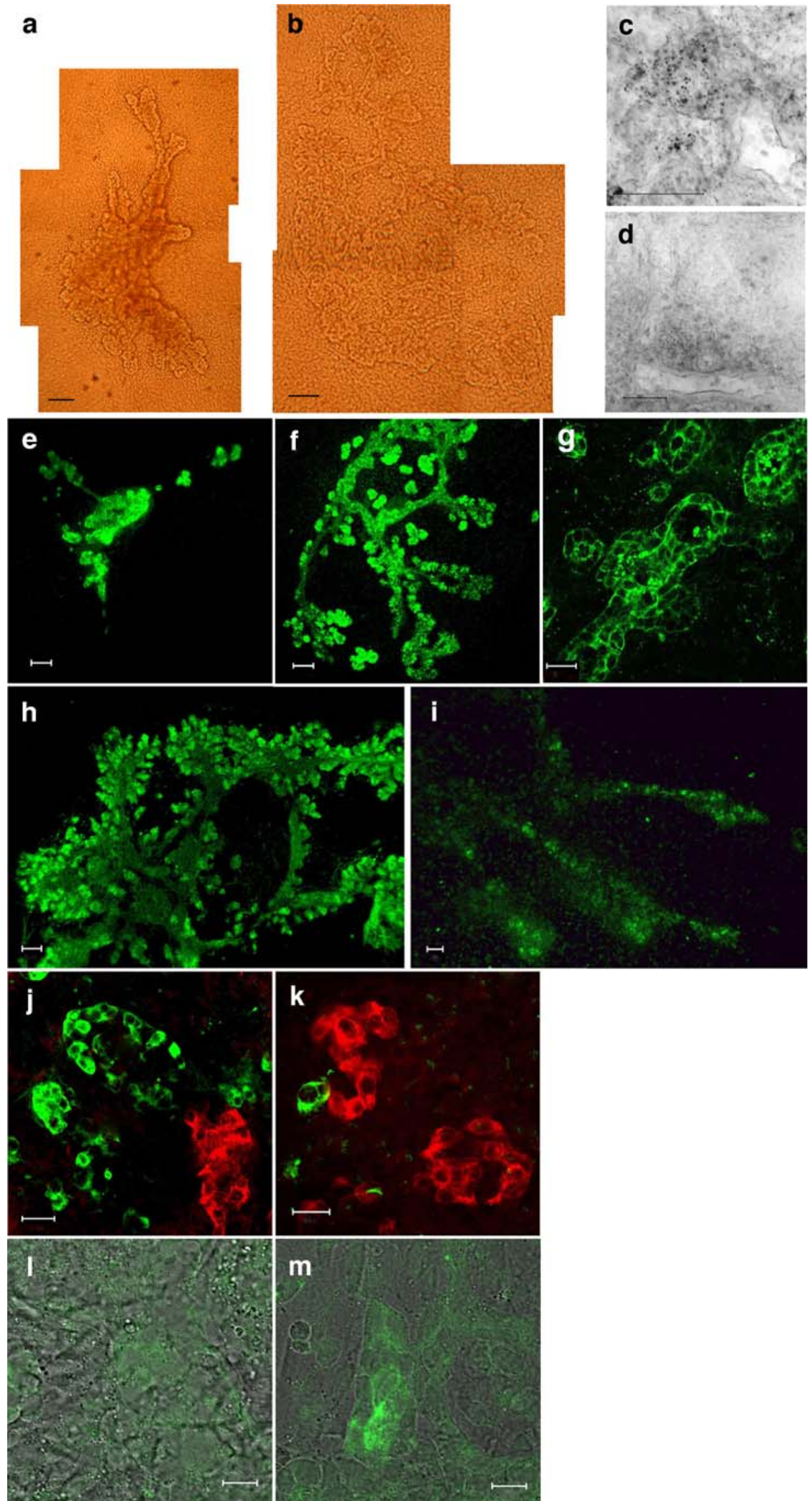
Effect of betacellulin on branching morphogenesis Since EGFR ligands can promote branching morphogenesis [5, $15,21]$, we investigated the effect of betacellulin on the branching of the pancreatic ductal network. During the first few days of culture the epithelium becomes an extended branched structure, which can be observed under transmitted light conditions (Fig. 2a). Addition of betacellulin increased branching and dilation of the incipient pancreatic ducts (Fig. 2b). CK19, a cytoskeletal protein, is present in the incipient ductal cells of the pancreas as well as in developing acini [22]. To confirm the increase in branching morphogenesis, we immunostained control and betacellulin-treated cultures with an anti-CK19 antibody. Betacellulin promoted branching morphogenesis based on the more extensive CK19 immunostaining (Fig. 2e-g). The differences in branching between the untreated and betacellulin-treated buds were quantified by measuring the length along the CK19-positive branches of the pancreatic ductal network. The results indicate that there is a 3.5 -fold increase in branching induced by betacellulin (Table 1). This implies a possible role for betacellulin in proliferation of the incipient ducts. To test this idea, we co-stained for the cell-proliferation marker phosphohistone-3 (PH3) with CK19 in control and betacellulin-treated samples. However, no co-localisation of PH3 with CK19-positive cells was observed at any of the four time-points examined (days 2, 3, 5 and 9), suggesting that proliferation of the ductal cells may take place prior to their becoming CK19positive (Fig. 3d,e). Instead, PH3-positive cells were mainly localised to the mesenchyme layer (Fig. 3f-h). Overall, betacellulin-treated samples contained increased numbers of PH3-positive cells compared with controls (Table 1).

Betacellulin reduces the amylase pancreatic population Amylase-expressing cells were present in the control pancreatic organ cultures at day 7 and the numbers had increased further by day 10 . The amylase-expressing cells were distributed around the periphery of the branches and were distinct from the endocrine cell populations (Fig. 2h,j). In contrast, the betacellulin-treated pancreatic tissue either completely lacked or had reduced numbers of amylaseexpressing cells (Fig. 2i,k). Under transmitted light, the zymogen granules, which store the exocrine enzymes, are clearly visible in the control (Fig. 2c) but not in the betacellulin-treated (Fig. 2d). Overall, betacellulin treatment reduced the number of amylase-positive cells (measured as the total area covered by amylase-positive cells) by 18.5 fold (Table 1). Western blot analysis confirmed this observation and showed the virtual absence of the amylase protein in betacellulin-treated samples (see Fig. 4). Furthermore, the pro-apoptotic protein caspase-3 was observed in acinar tissue of betacellulin-treated samples (Fig. 21,m),

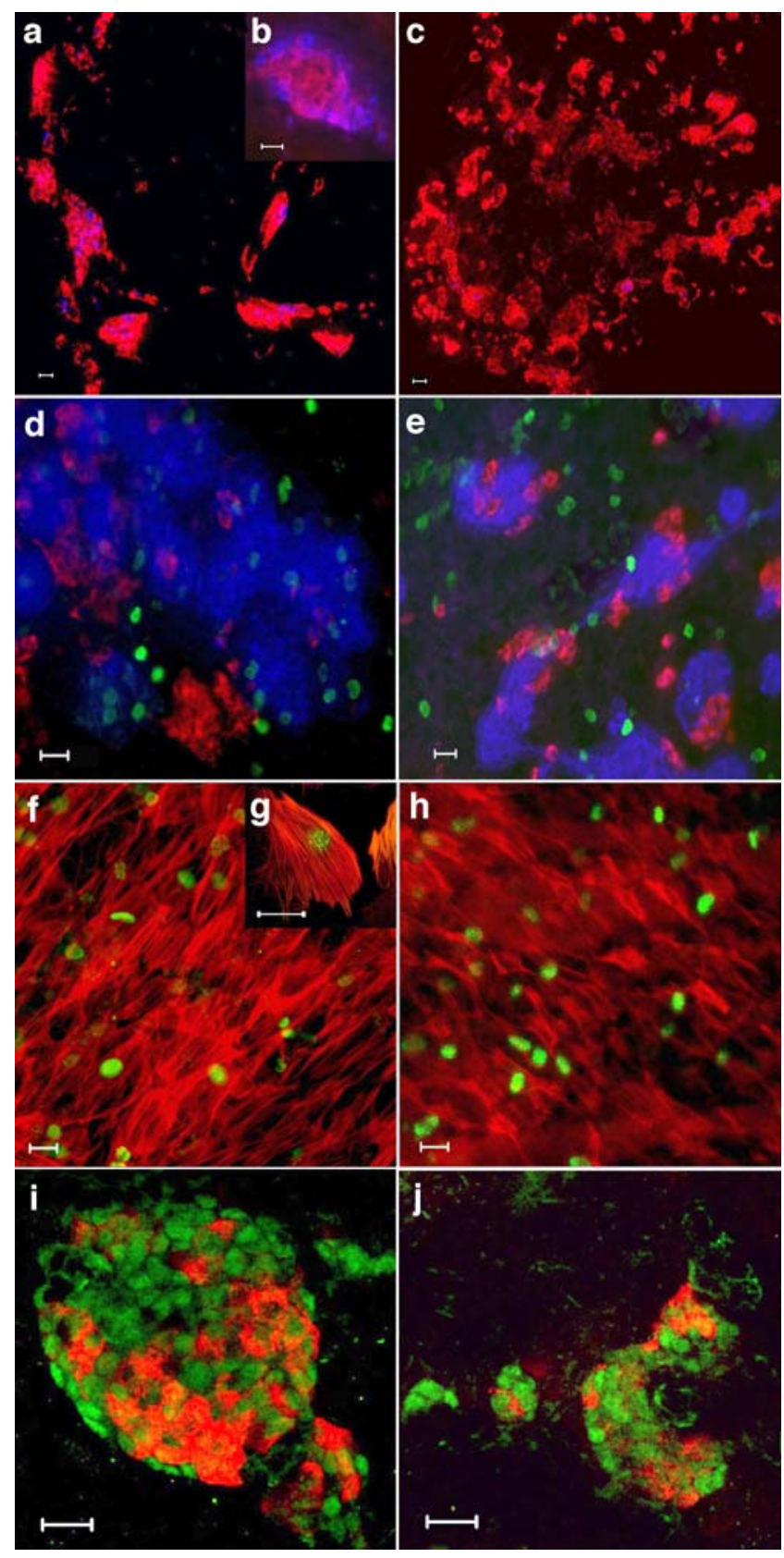

Fig. 3 Betacellulin regulates endocrine cell development. a-c Control $(\mathbf{a}, \mathbf{b})$ and betacellulin-treated (c) pancreatic buds after 9 days of treatment, immunostained for insulin (red) and glucagon (blue). Insulinpositive cells are more abundant in betacellulin-treated cultures compared with the controls. Treatment with betacellulin also resulted in a marked reduction in the number of glucagon-positive cells (blue). $\mathbf{d}, \mathbf{e}$ The increase in the number of beta cells in not due to an increase in cell division since there was little or no co-localisation of the proliferation marker PH3 (green) with either insulin (red) or CK19 (blue). f-h Instead PH3 (green) tended to co-localise with the mesenchymal cells stained for smooth muscle actin (red) in both control (f, g) and betacellulin-treated tissue (h). i, j Staining for Pax6 (green) and glucagon (red) shows the reduction in cells expressing Pax6 in betacellulin-treated samples (j) compared with the controls (i). Scale bars: a, c $100 \mu \mathrm{m} ; \mathbf{d}-\mathbf{f}, \mathbf{h}-\mathbf{j} 20 \mu \mathrm{m} ; \mathbf{b}, \mathbf{g} 10 \mu \mathrm{m}$. Pax6 transcription factor is expressed in progenitors for all pancreatic endocrine cells at some point and is necessary for alpha cell differentiation 
whereas it was much more difficult to find caspase-3positive cells in control buds.

Betacellulin promotes beta cell differentiation We investigated the effect of betacellulin on insulin-expressing endocrine cells. In both betacellulin-treated and untreated samples, after 10 days of culture, insulin-positive beta cells out-numbered any other endocrine cell type. However, the distribution and organisation of the beta cells were noticeably different between treated and untreated samples. In the untreated pancreatic buds, there were greater numbers of beta cells clumped together in large islet-like structures (Fig. 3a,b). In contrast, the betacellulin-treated buds had smaller clumps of beta cells that were dispersed throughout the organ culture (Fig. 3c). Additionally there were many individual cells or small islet-like cell clusters positive for insulin in the inter-islet regions and in close proximity to the ducts. Betacellulin induced an approximately threefold increase in the number of beta cells (measured as the total area covered by insulin-positive cells) with no significant change in cell size (Table 1). This observation was also confirmed by western blotting for insulin protein (Fig. 4). To test whether betacellulin increased the number of beta cells by increasing the proliferation of beta cells we costained for insulin and the proliferation marker PH3. Betacellulin did not affect the proliferation of insulinpositive beta cells and we found only a few insulin-positive cells co-staining with PH3 (Fig. 3d,e).

Effects of betacellulin on alpha cell differentiation In control samples, the classic islet morphology was preserved with glucagon-expressing cells present in the periphery and these cells outnumbered all other endocrine cell types except beta cells by day 10 of culture (Fig. 3a,b). However, in the betacellulin-treated samples, the number of glucagonpositive cells was reduced to just a few cells in each pancreas (Fig. 3c). Although glucagon protein was not detectable on the western blot, presumably because of its small size, the amount of the $18 \mathrm{kDa}$ pro-glucagon peptide from which glucagon is derived was much higher in the untreated than betacellulin-treated pancreatic samples (Fig. 4).

Effects of betacellulin on transcription factor expression Since there was no evidence for changes of proliferative rate underlying the observed effects of betacellulin on the epithelial cell types it is more likely that the effects are due to an alteration in lineage determination of precursor cells. To examine this we determined the expression of pancreatic transcription factors by immunohistochemistry. By day 2 of culture, both control and betacellulintreated samples showed high levels of Pdx1 (Fig. 5). Pdx1 is a homeodomain protein that is expressed in the entire pancreatic anlagen at early stages of development. Normally,
Pdx 1 expression declines during later embryonic stages in most of the pancreas but remains selectively expressed in the beta cells where it binds to and activates the insulin promoter. By day 5 of our cultures, the expression of Pdx 1 was indeed limited to insulin-positive cells in the untreated samples (data not shown).

In comparison, Pdx1 expression in the betacellulin-treated samples was not strictly confined to such insulin-positive cell clusters. Pdx1-positive cells in betacellulin-treated samples outnumbered all of the differentiated endocrine cells put together and the expression was more ubiquitous, extending to cell populations that might be acinar or ductal in nature. Pdx 1 protein was also higher in betacellulin-treated samples compared with controls (Fig. 4). Pdx1 expression is normally downregulated in non-beta cells during late embryonic development [23] and prolonged expression of

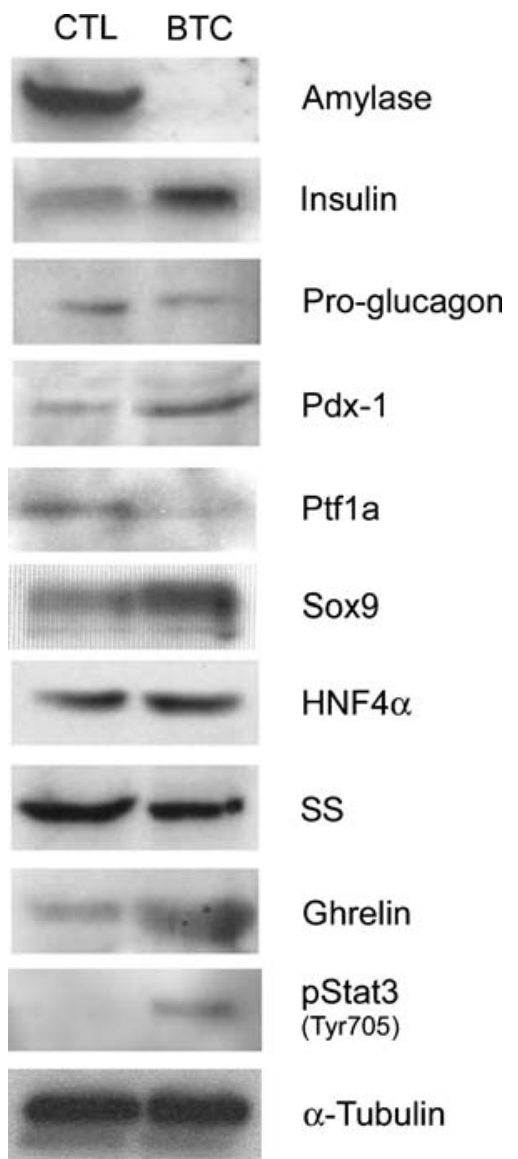

Fig. 4 The effects of betacellulin (BTC) treatment on the levels of pancreatic proteins. Protein was isolated from pancreatic buds cultured with and without betacellulin for 7 days. Following BTC treatment, there was an increase in the levels of the pancreatic hormones insulin and ghrelin, the transcription factors Pdx1 and Sox9, and the phosphorylated form (Tyr705) of the signalling molecule Stat3 (pStat3). The levels of the enzyme amylase, the immature form of the pancreatic hormone glucagon, and the exocrine promoting transcription factor Ptfla, were reduced. The levels of SS and HNF4 $\alpha$ remained the same in both control (CTL) and $B T C$-treated samples. $\alpha$-Tubulin was used as the loading control 

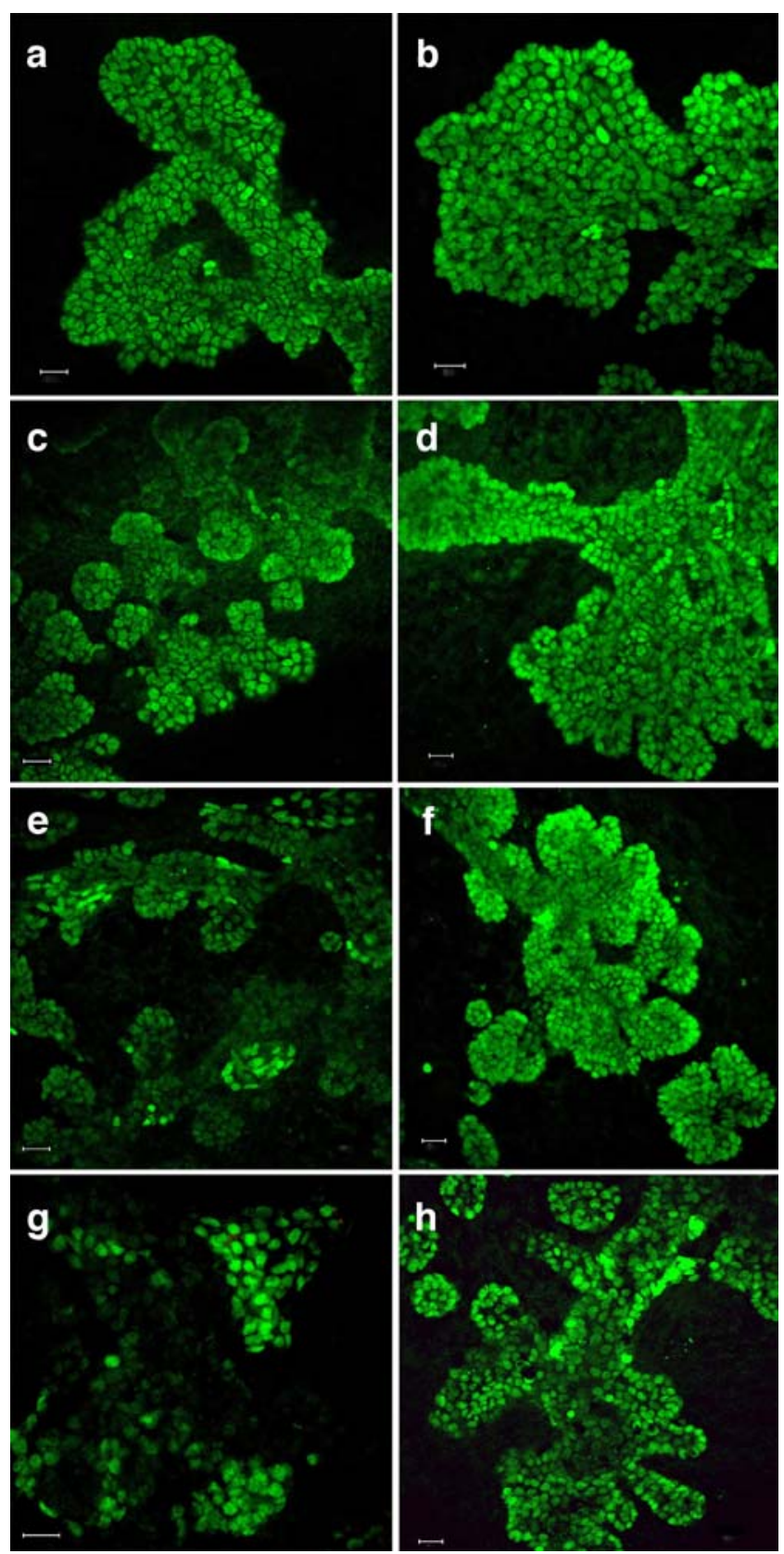

Fig. 5 Betacellulin induces the expression of Pdx1 in pancreatic cultures. Time course of expression of $\mathrm{Pdx} 1$ in control $(\mathbf{a}, \mathbf{c}, \mathbf{e}, \mathbf{g})$ and betacellulin-treated $(\mathbf{b}, \mathbf{d}, \mathbf{f}, \mathbf{h})$ cultures at 2 days $(\mathbf{a}, \mathbf{b}), 3$ days $(\mathbf{c}, \mathbf{d})$, 5 days $(\mathbf{e}, \mathbf{f})$ and 7 days $(\mathbf{g}, \mathbf{h})$ into culture with and without treatment. Buds were then fixed and immunostained for Pdx1 (green). In control cultures, Pdx1 becomes restricted to islet-like structures as development proceeds whereas in betacellulin-treated cultures there is much sustained widespread expression of Pdx 1 even at 7 days into culture (h). Scale bars: $20 \mu \mathrm{m}$

Pdx1 in the exocrine portion of the pancreas has been shown to cause acinar-to-ductal metaplasia mediated through activation of the signal transducer and activator of transcription-3 (Stat3) [24]. This is very similar to our observed effect, which is an increase of developing ducts relative to developing amylase-positive cells. So we were interested to know whether Stat3 is activated following betacellulin treatment. Previously, Stat3 has been shown to be activated in cells of human pancreatic cancers $[25,26]$ and in the metaplastic cells that form ductal cells [24]. When pancreases were treated with and without betacellulin for 7 days we found activated Stat3 (phosphorylated on Tyr705) in betacellulin-treated samples (Fig. 4). We did not observe any expression of phosphorylated Stat 3 in control samples at the time-points examined.

We also examined the expression of Pax6, which is essential for alpha cell differentiation. This transcription factor was much reduced in betacellulin-treated samples compared with untreated samples (Fig. 3i,j). Similarly the levels of the transcription factor Ptfla, which plays a vital role in early exocrine differentiation, was reduced following betacellulin treatment concomitant with the reduction in amylase expression (Fig. 4). On the other hand, hepatocyte nuclear factor (HNF) $4 \alpha$, which is not known to play a pivotal role in either alpha or beta cell differentiation, was expressed at similar levels in both treated and untreated samples (Fig. 4).

Effects of betacellulin on expression of pancreatic polypeptide (PP), somatostatin (SS) and ghrelin The effects of betacellulin on the PP cells, SS delta cells and the ghrelincontaining cells were also investigated (Fig. 6a-f). All these cell types were found in both the control and betacellulintreated samples, but in smaller numbers compared with beta and alpha cells.

PP cells were observed at a greater frequency in the untreated cultures than in the betacellulin-treated cultures (Table 1). Normally they are found clustered in or near the islets. In the betacellulin-treated samples, clusters of PP cells were absent and they were found mostly individually, in pairs or as triplets. The frequency of occurrence of delta cells within islets was similar in both betacellulin-treated and untreated cultures (Table 1). The differences in cell numbers for delta cells were relative to the size of the pancreatic buds with and without treatment. This observation was also confirmed by western blot analysis for the amounts of SS protein normalised to the total amount of protein (Fig. 4). Unlike PP cells, the delta cells did not cluster. Furthermore, as is typical in both treated and untreated samples, some of the SS-positive cells also co-expressed insulin (appears yellow on merged images).

Ghrelin was the least abundant islet hormone in the pancreatic organ cultures. However, the number of cells expressing ghrelin was higher following betacellulin treatment (Table 1) and western blotting showed that the betacellulin-treated samples had considerably higher levels of ghrelin protein compared with the untreated samples (Fig. 4). 

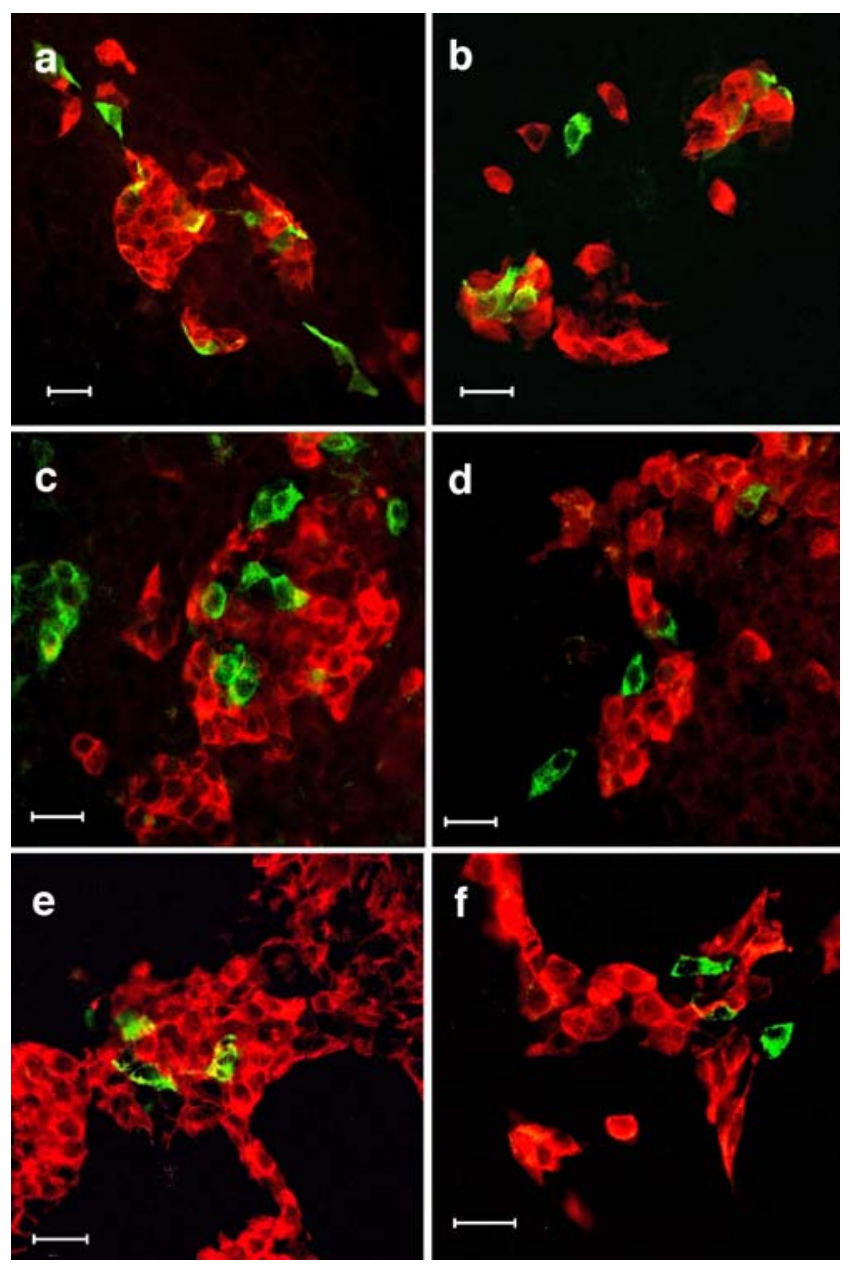

Fig. 6 Expression of the endocrine hormones, SS, PP and ghrelin in the embryonic pancreas. E11.5d embryonic pancreatic buds were cultured without $(\mathbf{a}, \mathbf{c}, \mathbf{e})$ or with $(\mathbf{b}, \mathbf{d}, \mathbf{f})$ betacellulin for 10 days as described in the "Materials and methods" section and then co-stained for SS (a, b), PP (c, d) or ghrelin (e, f) (green) and insulin (red). Note that since these cell types are rare and only found within islets, the pictures show high magnification images of only the areas with an abundance of insulin-positive cells for both controls and betacellulintreated samples. Scale bars: $20 \mu \mathrm{m}$

\section{Discussion}

In the present study we have extended previous studies [3, $21]$ to show that addition of exogenous betacellulin induces multiple effects on embryonic pancreatic development. Addition of betacellulin inhibits expression of amylase and glucagon, increases the expression of insulin and ghrelin and enhances branching morphogenesis in an in vitro model of murine embryonic pancreas. Betacellulinnull mice do not display any overt pancreatic defects, possibly due to compensation by other EGFR ligands [9]. Due to this functional redundancy within the EGFR ligand family, we added EGF or TGF $\alpha$ to pancreatic buds. Addition of EGF and TGF $\alpha$ promoted branching morpho- genesis and reduced amylase expression in cultured pancreatic buds as expected, but neither factor reduced the number of glucagon-positive cells (data not shown). Pancreases of EGFR-deficient mice show impaired ductal branching, suggesting that this phenotype is mediated, in part, by the activation of a common EGFR downstream signalling pathway, possibly the MEK/ERK pathway [21, 27]. However, in contrast to betacellulin and TGF $\alpha$ action, EGF reduced the number of insulin-positive beta cells (data not shown), as previously reported [28]. These observations may be explained by differences in receptor binding (EGF or TGF $\alpha$ bind with high affinity to ErbB1 but not to ErbB4 receptors, whereas betacellulin binds with high affinity to both receptors [29]) or through the differential activation of EGFR downstream signalling pathways [30]. This could explain why the effect of betacellulin is more pronounced and extends to multiple cell types compared with EGF or TGF $\alpha$ (Figs. 2, 3 and 6). Supporting this possibility is the observation that ubiquitous expression of betacellulin in vivo results in phenotypic changes in multiple cell types of various different organs [31].

To date, no study has examined the effects of betacellulin on branching morphogenesis in pancreatic explant cultures from E11.5d embryos. Using CK19 as a marker of ductal cells, we showed that betacellulin increases branching morphogenesis. The mechanism of betacellulin action on ductal morphogenesis is probably mediated by increased differentiation from epithelial progenitors rather than by increased proliferation. This is reminiscent of the transdifferentiation of acinar cells to ductal cells that has been observed in culture [32] and in transgenic mice overexpressing the gene for Pdx1 [24]. Consistent with this we find an increase in the level of activated Stat3.

It is also probable that betacellulin is delaying or suppressing differentiation of the primordial duct-like epithelium into amylase-positive cells as a result of the continued expression of the gene encoding Pdx1. It is known that mesenchymal factors such as follistatin can regulate the proportion of exocrine and endocrine cell types [33]. We did attempt studies on isolated epithelium but found that removing the mesenchyme resulted in no growth or branching of the isolated epithelium and few insulinexpressing cells were present even after a week of culture (results not shown). However, we observed a reduction in the number of amylase-positive cells in the presence of mesenchymal proliferation. Since the genes for the ErbB1 and ErbB4 receptors are differentially expressed in both the mesenchyme and the epithelium, it is possible that the effect of betacellulin on both tissue types is independent rather than the change in amylase being brought about via a paracrine action emanating from the mesenchyme. Therefore the reduction in amylase-positive cells might also be mediated through apoptosis, since persistent upregulation 
of Pdx 1 has previously been shown to destroy pancreatic acinar cells by apoptosis [34]. It has also been observed that acinar apoptosis is associated with ductal proliferation after duct ligation [35]. We saw an upregulation of activated caspase-3, after betacellulin treatment and a concurrent decrease in the level of the transcription factor Ptfla, which is necessary for early exocrine determination. The combined data suggest that the effect of betacellulin on the exocrine cells might in fact act at two different levels. First, around the time when the exocrine and ductal fates are determined by promoting progenitors towards a ductal fate, and second, by inducing apoptosis of already differentiated amylase-positive cells or promoting their transdifferentiation into ductal cells much later on. One hypothesis to explain the pro-apoptotic action of betacellulin compared with other receptor tyrosine kinase ligands (e.g. EGF) is that it is acting through a different receptor or post-receptor events [29].

In one previous study no change in the number of amylase-expressing cells was seen after betacellulin treatment; however, the time of treatment was only half of that used by ourselves [5].

We show by immunofluorescence microscopy and western blotting, that betacellulin enhances the number of insulin-positive beta cells and the overall levels of insulin in the developing pancreas. Betacellulin is reported to regulate the proportion of beta cells in other systems $[5,11,14]$. Although our study agrees with previous observations showing an increase in the number of beta cells after betacellulin treatment [5], we show a more profound effect, which may again be due to the longer duration of the treatment used. Three mechanisms might explain the increase in the number of beta cells. The additional beta cells could arise by: (1) the proliferation of existing beta cells; (2) the differentiation of endocrine precursor cells into beta cells rather than other endocrine cells; or (3) the transdifferentiation of exocrine cell types into beta cells.

The first possibility can be ruled out because if the extra beta cells did arise due to the proliferation of existing beta cells we would observe larger islets and an increased number of cells that show co-localisation for $\mathrm{PH} 3$ and insulin.

Huotari et al. [5] described the modulation of endocrine lineages by different growth factors. In the absence of EGFR signalling, differentiation followed a default pathway towards alpha and PP cells. In the present study, the reduction in the number of alpha and PP cells in the presence of betacellulin suggests that betacellulin might be influencing the fate choice made by the early endocrine precursor cells and shifting the balance towards a beta cell phenotype. All pancreatic endocrine cell types initially arise from common multipotent precursor cells that express Pdx1 but the continued expression of $\mathrm{Pdx} 1$ is only necessary for the development of beta cells and not other endocrine cell types [36]. The widespread late Pdx1 expression seen in the betacellulin-treated pancreases might therefore bias the commitment of endocrine precursors toward beta cells. Furthermore, the betacellulin-induced downregulation in expression of other transcription factors such as the alpha cell determinant Pax6 would ensure that the precursor cells do not differentiate down this lineage (Fig. 3). Cell lineage studies have localised the endocrine progenitor cells close to the primitive ducts of the fetal pancreas [37]. The Pdx1 expression that is not restricted to the islets is seen in these regions after betacellulin treatment (Fig. 5).

Ogata et al. [38] showed that betacellulin in combination with activin A can induce the differentiation of monolayer cultures of rat neonatal ductal cells to beta cells. Threedimensional pseudoislets can be formed by making suspensions of activin A- and betacellulin-treated cells and culturing with just betacellulin alone. The process whereby endocrine tissue is generated from ductal epithelium is termed nesidioblastosis [39]. It has been reported in many experimental models and in clinical pathology [40]. During proliferation, duct cells have the potential to lose their ductal phenotype and revert back to multipotent cells [41]. This is achieved by the ductal cells expressing Pdx1, differentiating into beta cells, then migrating away from the ducts and clustering to form islets [35]. The possibility of duct cell derived beta cells in this manner could explain the enhanced number of Pdx1-expressing cells in the ducts, the presence of islet-like cell clusters and the insulin-positive cells found in isolation. So, betacellulin is probably influencing beta cell formation both by increasing the proportion of endocrine cells and by increasing the proportion of endocrine precursors that become beta cells.

The present study shows that betacellulin can enhance branching morphogenesis of the embryonic pancreatic branching network and increase beta cell mass. We suggest that these changes are largely brought about by prolonged ubiquitous expression of Pdx1. Elevated Pdx1 expression suppresses early differentiation of the endodermal pancreatic precursor tissue and enhances branching and formation of beta cells. This makes betacellulin a promising candidate for stimulating beta cell regeneration in vivo, or increasing the proportion of beta cells in vitro in situations where beta cell differentiation is being sought with a view to transplantation.

Acknowledgements The authors wish to thank E. B. Lane (University of Dundee) for generously providing the anti-CK19 antibody. This work was supported by a Universities UK Overseas Research Studentship and the Department of Biology and Biochemistry, University of Bath (S. Thowfeequ). Financial support was also provided by the Biotechnology and Biological Sciences Research Council, Medical Research Council and the Wellcome Trust.

Duality of interest None of the authors has any financial interest in this work. 


\section{References}

1. Shapiro AM, Geng Hao E, Lakey JR, Finegood DT, Rajotte RV, Kneteman NM (2002) Defining optimal immunosuppression for islet transplantation based on reduced diabetogenicity in canine islet autografts. Transplantation 74:1522-1528

2. Naftanel MA, Harlan DM (2004) Pancreatic islet transplantation. PLoS Med 1:e58 (quiz e75)

3. Bonner-Weir S, Weir GC (2005) New sources of pancreatic betacells. Nat Biotechnol 23:857-861

4. Zalzman M, Anker-Kitai L, Efrat S (2005) Differentiation of human liver-derived, insulin-producing cells toward the beta-cell phenotype. Diabetes 54:2568-2575

5. Huotari MA, Miettinen PJ, Palgi J et al (2002) ErbB signaling regulates lineage determination of developing pancreatic islet cells in embryonic organ culture. Endocrinology 143:4437-4446

6. Dunbar AJ, Goddard C (2000) Structure-function and biological role of betacellulin. Int J Biochem Cell Biol 32:805-815

7. Sasada R, Ono Y, Taniyama Y, Shing Y, Folkman J, Igarashi K (1993) Cloning and expression of cDNA encoding human betacellulin, a new member of the EGF family. Biochem Biophys Res Commun 190:1173-1179

8. Miyagawa J, Hanafusa O, Sasada R et al (1999) Immunohistochemical localization of betacellulin, a new member of the EGF family, in normal human pancreas and islet tumor cells. Endocr J 46:755-764

9. Jackson LF, Qiu TH, Sunnarborg SW et al (2003) Defective valvulogenesis in HB-EGF and TACE-null mice is associated with aberrant BMP signaling. EMBO J 22:2704-2716

10. Mashima H, Ohnishi H, Wakabayashi K et al (1996) Betacellulin and activin A coordinately convert amylase-secreting pancreatic AR42J cells into insulin-secreting cells. J Clin Invest 97:1647-1654

11. Demeterco C, Beattie GM, Dib SA, Lopez AD, Hayek A (2000) A role for activin A and betacellulin in human fetal pancreatic cell differentiation and growth. J Clin Endocrinol Metab 85:3892-3897

12. Li L, Seno M, Yamada H, Kojima I (2003) Betacellulin improves glucose metabolism by promoting conversion of intraislet precursor cells to beta-cells in streptozotocin-treated mice. Am J Physiol Endocrinol Metab 285:E577-E583

13. Kojima H, Fujimiya M, Matsumura K et al (2003) NeuroDbetacellulin gene therapy induces islet neogenesis in the liver and reverses diabetes in mice. Nat Med 9:596-603

14. Li L, Seno M, Yamada H, Kojima I (2001) Promotion of beta-cell regeneration by betacellulin in ninety percent-pancreatectomized rats. Endocrinology 142:5379-5385

15. Sakurai H, Tsukamoto T, Kjelsberg CA, Cantley LG, Nigam SK (1997) EGF receptor ligands are a large fraction of in vitro branching morphogens secreted by embryonic kidney. Am J Physiol 273:F463-F472

16. Kim SK, Hebrok M (2001) Intercellular signals regulating pancreas development and function. Genes Dev 15:111-127

17. Percival AC, Slack JM (1999) Analysis of pancreatic development using a cell lineage label. Exp Cell Res 247:123-132

18. Shen CN, Seckl JR, Slack JM, Tosh D (2003) Glucocorticoids suppress beta-cell development and induce hepatic metaplasia in embryonic pancreas. Biochem J 375:41-50

19. Li WC, Horb ME, Tosh D, Slack JM (2005) In vitro transdifferentiation of hepatoma cells into functional pancreatic cells. Mech Dev 122:835-847

20. Jiang FX, Harrison LC (2005) Laminin-1 and epidermal growth factor family members co-stimulate fetal pancreas cell proliferation and colony formation. Differentiation 73:45-49

21. Miettinen PJ, Huotari M, Koivisto T et al (2000) Impaired migration and delayed differentiation of pancreatic islet cells in mice lacking EGF-receptors. Development 127:2617-2627
22. Bouwens L, Braet F, Heimberg H (1995) Identification of rat pancreatic duct cells by their expression of cytokeratins 7,19 , and 20 in vivo and after isolation and culture. J Histochem Cytochem $43: 245-253$

23. Stoffers DA, Heller RS, Miller CP, Habener JF (1999) Developmental expression of the homeodomain protein IDX-1 in mice transgenic for an IDX-1 promoter/lacZ transcriptional reporter. Endocrinology 140:5374-5381

24. Miyatsuka T, Kaneto H, Shiraiwa $T$ et al (2006) Persistent expression of PDX-1 in the pancreas causes acinar-to-ductal metaplasia through Stat3 activation. Genes Dev 20:1435-1440

25. Greten FR, Weber CK, Greten TF et al (2002) Stat3 and NFkappaB activation prevents apoptosis in pancreatic carcinogenesis. Gastroenterology 123:2052-2063

26. Scholz A, Heinze S, Detjen KM et al (2003) Activated signal transducer and activator of transcription 3 (STAT3) supports the malignant phenotype of human pancreatic cancer. Gastroenterology 125:891-905

27. Rescan C, Le Bras S, Lefebvre VH et al (2005) EGF-induced proliferation of adult human pancreatic duct cells is mediated by the MEK/ERK cascade. Lab Invest 85:65-74

28. Cras-Méneur C, Elghazi L, Czernichow P, Scharfmann R (2001) Epidermal growth factor increases undifferentiated pancreatic embryonic cells in vitro: a balance between proliferation and differentiation. Diabetes 50:1571-1579

29. Jones JT, Akita RW, Sliwkowski MX (1999) Binding specificities and affinities of EGF domains for ErbB receptors. FEBS Lett 447:227-231

30. Saito T, Okada S, Ohshima K et al (2004) Differential activation of epidermal growth factor (EGF) receptor downstream signaling pathways by betacellulin and EGF. Endocrinology 145:42324243

31. Schneider MR, Dahlhoff M, Herbach N et al (2005) Betacellulin overexpression in transgenic mice causes disproportionate growth, pulmonary hemorrhage syndrome, and complex eye pathology. Endocrinology 146:5237-5246

32. Rooman I, Heremans Y, Heimberg H, Bouwens L (2000) Modulation of rat pancreatic acinoductal transdifferentiation and expression of PDX-1 in vitro. Diabetologia 43:907-914

33. Miralles F, Czernichow P, Scharfmann R (1998) Follistatin regulates the relative proportions of endocrine vs exocrine tissue during pancreatic development. Development 125:1017-1024

34. Heller RS, Stoffers DA, Bock T et al (2001) Improved glucose tolerance and acinar dysmorphogenesis by targeted expression of transcription factor PDX-1 to the exocrine pancreas. Diabetes 50:1553-1561

35. Abe K, Watanabe S (1995) Apoptosis of mouse pancreatic acinar cells after duct ligation. Arch Histol Cytol 58:221-229

36. Jensen J (2004) Gene regulatory factors in pancreatic development. Dev Dyn 229:176-200

37. Ogata T, Li L, Yamada S et al (2004) Promotion of beta-cell differentiation by conophylline in fetal and neonatal rat pancreas. Diabetes 53:2596-2602

38. Ogata T, Park KY, Seno M, Kojima I (2004) Reversal of streptozotocin-induced hyperglycemia by transplantation of pseudoislets consisting of beta cells derived from ductal cells. Endocr J 51:381-386

39. Laguesse E (1893) Sur la formation des îlots de Langerhans dans le pancréas. Comptes Rendus Séances Soc Biol Fil 45:819-820

40. Kerr-Conte J, Pattou F, Lecomte-Houcke M et al (1996) Ductal cyst formation in collagen-embedded adult human islet preparations. A means to the reproduction of nesidioblastosis in vitro. Diabetes 45:1108-1114

41. Bonner-Weir S, Taneja M, Weir GC et al (2000) In vitro cultivation of human islets from expanded ductal tissue. Proc Natl Acad Sci U S A 97:7999-8004 